\title{
Analysis of Skeletal Microstructure with Clinical Multislice CT
}

\author{
Joel Petersson ${ }^{1,2}$, Torkel Brismar ${ }^{3}$, and Örjan Smedby ${ }^{1,2,4}$ \\ ${ }^{1}$ Center for Medical Image Science and Visualization (CMIV), Linköping University, \\ Sweden \\ ${ }^{2}$ Department of Science and Technology (ITN), Linköping University, Sweden \\ ${ }^{3}$ Department of Radiology, Karolinska University Hospital Huddinge, Sweden \\ ${ }^{4}$ Department of Medicine and Care (IMV), Linköping University, Sweden*
}

\begin{abstract}
In view of the great effects of osteoporosis on public health, it would be of great value to be able to measure the three-dimensional structure of trabecular bone in vivo as a means to diagnose and quantify the disease. The aim of this work was to implement a method for quantitative characterisation of trabecular bone structure using clinical CT.

Several previously described parameters have been calculated from volumes acquired with a 64 -slice clinical scanner. Using automated region growing, distance transforms and three-dimensional thinning, measures describing the number, thickness and spacing of bone trabeculae was obtained. Fifteen bone biopsies were analysed. The results were evaluated using micro-CT as reference.

For most parameters studied, the absolute values did not agree well with the reference method, but several parameters were closely correlated with the reference method. The shortcomings appear to be due to the low resolution and high noise level. However, the high correlation found between clinical CT and micro-CT measurements suggest that it might be possible to monitor changes in the trabecular structure in vivo.
\end{abstract}

\section{Introduction}

The high prevalence and great societal costs of osteoporosis necessitate methods to quantify the disease in vivo, in particular when evaluating treatment. Currently, such quantification is most commonly made with dual energy X-ray absorptiometry (DXA) [1], a method which measures the mineral content of bone but fails to describe its trabecular structure. In post-mortem specimens, several parameters describing the structure in terms such as number, thickness and spacing of bone trabeculae have been identified, initially from micrographs of $2 \mathrm{D}$ sections, but later also from micro computed tomography [2]. For clinical as well as research purposes, it would be very attractive to be able to make similar measurements with a modality that can be used in vivo, such as clinical computed tomography (CT).

\footnotetext{
* The authors are thankful to Andres Laib for providing micro-CT data, to Ingela Nyström for valuable methodological suggestions and to Johanna Petersson for technical assistance.
} 
The aim of this work was to ascertain whether useful estimates of parameters describing bone structure in specimens can be obtained with clinical CT, by implementing such a method, testing it in vitro and correlating it to the reference method micro-CT.

\section{Material and Methods}

All the methods described in this section was implemented using MATLAB on a standard PC with two $1.7 \mathrm{GHz}$ processors and $2 \mathrm{~GB}$ of RAM.

\section{$2.1 \quad$ Samples}

The samples consisted of 15 bone biopsies from the radius. The biopsies were approximately cubic with a side of $10 \mathrm{~mm}$. Each cube included a thin slab of cortical bone on one side to facilitate orientation. One of the samples is shown in Figure 1] When imaged with clinical CT, the bone sample was placed in a test tube filled with water, and the tube was then placed in the centre of a paraffin cylinder with a diameter of approximately $10 \mathrm{~cm}$, representing soft tissue to simulate measurement in vivo.

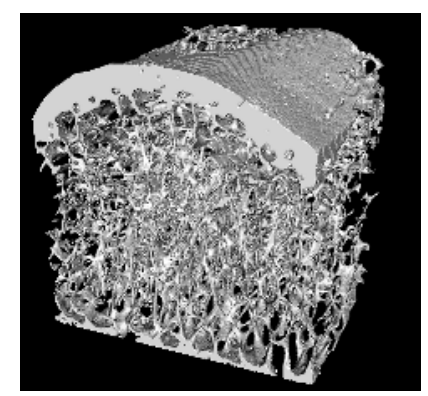

Fig. 1. One of the trabecular bone biopsies reconstructed from micro-CT data

\subsection{Data Acquisition}

The clinical CT used was a 64-slice LightSpeed VCT (GE Medical Systems, Milwaukee, WI, USA). The acquired data were reconstructed using the kernel called "boneplus". Acquisition parameters are shown in Table11 Micro-CT data were acquired with a small desktop CT used for analysing biopsies and other specimens ( $\mu \mathrm{CT} 40$; SCANCO Medical AG, Bassersdorf, Switzerland). The specimens can have a maximum diameter of $36 \mathrm{~mm}$ and a maximum length of $80 \mathrm{~mm}$. The micro-CT volumes were acquired at SCANCO using a tube voltage of 70 $\mathrm{kVp}$ and an isotropic resolution of $20 \mu \mathrm{m}$. The micro-CT data were used as a reference to evaluate the clinical CT data.

A cube, approximately $8 \mathrm{~mm}$ in side, containing only trabecular bone was extracted from each dataset for analysis. The coordinate system was placed with the z-axis along the axial sections and the y-axis along the cortical slab. 
Table 1. Clinical CT acquisition protocol

\begin{tabular}{ll}
\hline Property & Value \\
\hline Voxel size & $0.188 \times 0.188 \times 0.1 \mathrm{~mm}$ \\
Slice thickness & $0.625 \mathrm{~mm}$ \\
Tube voltage & $120 \mathrm{kV}$ \\
Effective mAs & 130 \\
Field of view & $96 \mathrm{~mm}$ \\
Matrix & $512 \times 512$ \\
Convolution kernel Boneplus \\
\hline
\end{tabular}

\subsection{Segmentation}

To obtain approximately isotropic voxels, each pair of sections was averaged into one single section in the volumes acquired with clinical $\mathrm{CT}$, thus obtaining a resolution of $0.188 \times 0.188 \times 0.2 \mathrm{~mm}$. Due to the averaging, some of the noise was also eliminated. The trabecular structure was then extracted using a modified version of the automated $3 \mathrm{D}$ region growing algorithm (ARG), developed by Revol-Muller et al. 3].

The ARG algorithm iterates a minimum variance region growing algorithm, [4, until the optimal segmentation is achieved according to an assessment function. Several assessment functions are proposed in [3]; in this study the $D_{G L^{-}}$ function was used. The function is region-based and measures the distance between the grey level function and a step function based on the average level in the segmented region and background respectively.

The original ARG method was developed for bimodal histograms. However, the noise level of clinical CT was too high and there was no minimum between the distributions corresponding to bone and water respectively. Instead, the following method was used. The seed region, i.e. the first undersegmented volume, was created by thresholding at a level obtained by adding to the histogram maximum a constant large enough to ensure undersegmentation. The lower limit of the variance interval was chosen as the variance of the voxels corresponding to the undersegmented volume. The upper limit was chosen as the lower limit multiplied by a constant.

For the micro-CT data, the histogram was bimodal and the two distributions could be approximated with two gaussian functions. The intersection between the two gaussians was used as a threshold. The variance of the thresholded volume was used as the centre of the variance interval. The length of the interval was chosen by multiplying the centre variance by a constant. And the initial seed region was obtained by performing an erosion of the thresholded volume.

There was no need for individual thresholding of the volumes, and thus the segmentation could be performed in a completely automated mode.

\subsection{Thinning}

The thinning algorithm is a morphologic operation designed to erode a binary volume without changing its topology, i.e. the number of cavities, tunnels and 
components are preserved. The segmented structure was thinned to a unit-wide skeleton representing the midline of the structure. The method implemented in this study was a parallel algorithm based on the removal of simple points [5]. Using a thinning algorithm to estimate the midline, rather than, e.g., a centres-of-maximal-balls algorithm, guarantees that the skeleton is unit-wide with preserved topology. This makes it is possible to estimate parameters such as the number of nodes per volume (Tb.Nd) and termini per volume (Tb.Tm). An example of the thinning process is shown in Figure 2 .

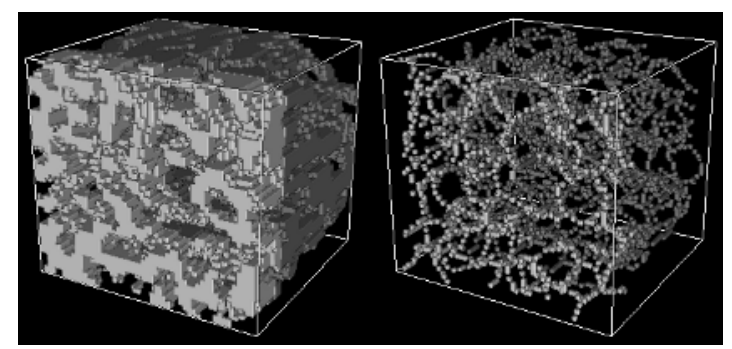

Fig. 2. Left: Result of the segmentation of a trabecular bone cube acquired with clinical CT. Right: Result of the thinning process applied to the left image. The first ten voxel layers are brighter for clarity.

\subsection{Distance Transforms}

When a distance transform is applied to a binary region, the value in each background voxel is replaced by its distance to the closest component voxel. In this study, the exact Euclidean distance transform was used whenever feasible. However, for the centres-of-maximal-balls algorithm described below, which requires a locally derived distance map, the faster chamfer 3-4-5 distance transform [6] was used instead.

\subsection{Estimation of Mean Distance}

Two methods to estimate the mean distance were used, local maxima (LM), and centres of maximal balls (CMB).

Local Maxima (LM). The local maximum is here defined by:

Definition 1. Let $D T_{E u c}$ be the Euclidean distance transform of a binary volume. A voxel $p \in\left\{D T_{\text {Euc }}>0\right\}$ is considered a local maximum if all its 26neighbours, $q \in N_{e}^{26}(p)$, has a distance label less or equal to the distance label of $q$ :

$$
p \in L M \leftrightarrow D T_{E u c}(p) \geq D T_{E u c}(q) \forall q \in N_{e}^{26}(p)
$$

The mean distance is then estimated by averaging the distance labels of the detected LM. 
Centres of Maximal Balls (CMB). Each value in a distance map can be considered as the radius of a sphere, the surface of which touches at least one object in the corresponding binary image. The centres-of-maximal-balls algorithm detects all spheres not completely covered by another sphere. As the CMB algorithm generalised to 3D [7] requires a locally defined distance transform, the chamfer 3-4-5 distance was used. The mean distance is then estimated by averaging the distance labels of the detected CMB.

\subsection{Calculating Structural Parameters}

All the parameters are calculated directly from the three-dimensional data. No models describing the shape of trabeculae were assumed. The parameters studied have been standardized by Parfitt et al. 2] except for Tb.Nd and Tb.Tm, which have been generalized to 3D in this study. Some of the parameters were estimated using both local maxima and centres of maximal balls. To distinguish these, LM or $\mathrm{CMB}$ have been added after the parameter abbreviation.

The bone fraction $(\mathrm{BV} / \mathrm{TV})$ was calculated as the number of voxels identified as bone in the segmentation divided by the total number of voxels in the volume.

Trabecular separation (Tb.Sp LM and Tb.Sp CMB) is the mean distance between the borders of the segmented trabeculae, calculated using local maxima and centres of maximal balls, respectively. The parameter is illustrated in Figure 3.

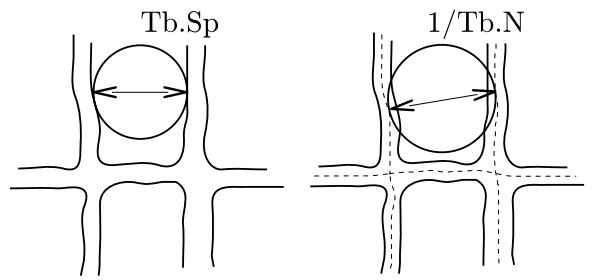

Fig. 3. The definition of Tb.Sp and Tb.N respectively. The measure is threedimensional but shown as a two-dimensional section for clarity.

Trabecular number (Tb.N) is the inverse of the mean spacing between the midlines of the trabeculae. The midlines of the trabeculae are estimated using the thinned structure. The spacing is calculated using both LM and CMB. The difference between T.Sp and Tb.N is illustrated in Figure 3 .

The thickness of the trabeculae ( $\mathrm{Tb} . \mathrm{Th}$ ) was estimated by first calculating the Euclidean distance transform of the complement of the segmented volume. Thus, each voxel value in the trabeculae is replaced with the distance to the nearest background voxel. The distance labels along the thinned structure are then extracted and averaged to estimate the mean thickness.

Nodes per volume (Tb.Nd) is the number of trabecular intersections per volume. A node is identified in the thinned structure as a voxel with at least three 
neighbours in its 26 -neighbourhood, detected nodes within a $5 \times 5 \times 5$-region was treated as one. The total number of nodes is then divided by the total volume.

A terminus is a free end of a trabecula. The number of termini per volume is denoted by $\mathrm{Tb}$.Tm. This parameter is also calculated from the thinned structure as the number of voxels with only one neighbour in its 26-neighbourhood divided by the total volume.

To study dependence on orientation, the mean intercept length (MIL) was calculated in the three main directions using a three-voxel matching kernel. The kernel consists of two one-voxels and one zero-voxel. The kernel is traversed through the volume and intersections between bone and background are counted. The total traversed length is then divided by the number of water-to-bone intersections.

\section{Results}

The relationship between parameters estimated from clinical CT and microCT data is illustrated in Table 2. The results show considerable overestimation of thickness and distance parameters such as Tb.Th and Tb.Sp with clinical CT. Consequently, the parameter Tb.N, which is the inverse of the trabecular spacing, is underestimated. When the correlation coefficients between clinical CT and micro-CT were calculated, however, they were all above 0.7 , except for Tb.Th and Tb.Tm.

Table 2. Results from clinical CT compared to micro-CT

\begin{tabular}{llll}
\hline & $\begin{array}{l}\text { Clinical CT } \\
\text { mean } \pm \text { SD }\end{array}$ & $\begin{array}{l}\text { Micro-CT } \\
\text { mean } \pm \text { SD }\end{array}$ & r $(95 \%$ confidence limits $)$ \\
\hline Tb.Sp LM $[\mathrm{mm}]$ & $0.797 \pm 0.049$ & $0.683 \pm 0.126$ & $0.89(0.71 ; 0.96)$ \\
Tb.Sp CMB $[\mathrm{mm}]$ & $0.830 \pm 0.131$ & $0.618 \pm 0.128$ & $0.77(0.42 ; 0.92)$ \\
Tb.N LM $\left[\mathrm{mm}^{-1}\right]$ & $0.677 \pm 0.038$ & $1.151 \pm 0.167$ & $0.77(0.43 ; 0.92)$ \\
Tb.N CMB $\left[\mathrm{mm}^{-1}\right]$ & $0.795 \pm 0.071$ & $1.357 \pm 0.223$ & $0.81(0.51 ; 0.93)$ \\
Tb.Th Euc $[\mathrm{mm}]$ & $0.633 \pm 0.016$ & $0.128 \pm 0.015$ & $0.61(0.14 ; 0.85)$ \\
BV/TV $[1]$ & $0.424 \pm 0.063$ & $0.095 \pm 0.033$ & $0.93(0.80 ; 0.98)$ \\
Tb.Nd $\left[\mathrm{mm}^{-3}\right]$ & $0.296 \pm 0.049$ & $4.919 \pm 1.778$ & $0.74(0.37 ; 0.91)$ \\
Tb.Tm $\left[\mathrm{mm}^{-3}\right]$ & $0.147 \pm 0.025$ & $0.792 \pm 0.248$ & $0.08(-0.45 ; 0.57)$ \\
MILx $[\mathrm{mm}]$ & $3.068 \pm 0.468$ & $2.423 \pm 0.894$ & $0.83(0.55 ; 0.94)$ \\
MILy $[\mathrm{mm}]$ & $2.445 \pm 0.397$ & $2.026 \pm 0.638$ & $0.80(0.48 ; 0.93)$ \\
MILz $[\mathrm{mm}]$ & $5.140 \pm 0.708$ & $3.470 \pm 1.039$ & $0.76(0.41 ; 0.92)$ \\
\hline
\end{tabular}

\section{Discussion}

In this study, a method for analysing trabecular structure was implemented and tested in bone samples. The results indicate that most of the parameters used for characterising trabecular bone in micro-CT can also be calculated for specimens imaged with clinical $\mathrm{CT}$, with a result that is closely correlated to the reference 
method, although yielding large systematic errors. Only for trabecular thickness and number of termini, which seem to be most sensitive to low resolution, correlation coefficients below 0.7 were obtained.

A major reason for choosing the ARG segmentation was the possibility to automate the algorithm. Another consequence of the region growing is that small isolated structures are excluded. Simple thresholding and removal of small components would probably create similar result. However, the choice of threshold is not obvious and the results would depend on the individual selecting it.

The advantage of using a thinning algorithm is that the result is a one voxel wide structure. Often, the CMB algorithm is used to approximate midlines, which may result in Tb.N being biased due to the fact that the resulting $\mathrm{CMB}$ region is not one voxel wide. By using the thinned structure instead of LM or $\mathrm{CMB}$ for the Tb.Th calculations, the thickness is not biased by volume. Instead, Tb.Th is weighted by length which is more intuitive when measuring an elongated object.

Unfortunately, the attempt to use the resulting skeleton to estimate the number of nodes and ends resulted in a resolution dependent method. A thicker structure, in number of voxels, results in more spurious branching [5]. Future research will address this matter.

It is not completely clear how the measurements should be averaged. Now all detected LM or CMB are averaged. In particular with CMB there are more voxels detected in larger holes. This can be an advantage, as the measures are then to some degree weighted by volume. However, if volume weighting is desired, then the method presented by Hildebrand and Ruegsegger [8] is preferable since each voxel in the background is set to the value of the CMB that covers it. It is not obvious how this could be performed for LM, since the LM spheres do not cover the entire volume of background as CMB do.

The Tb.Sp measures show an overestimation due to partial volume effects, leaving the thinnest trabeculae undetected. Tb.N shows an underestimation instead since it is a measure of the number of trabeculae per length. Thus, with undetected trabeculae or with several trabeculae treated as one, the result is fewer trabeculae. Although the absolute values are not correct, the correlation is surprisingly strong for both Tb.Sp and Tb.N. Consistently using the technique tested in this report might therefore enable reliable detection of changes in these parameters in longitudinal studies, provided that it can easily be transferred to use in vivo.

Tb.Th shows a considerable overestimation and weak correlation, implying that the resolution is too low to correctly represent this aspect of trabecular structure. Similar results have previously been found by e.g. Laib and Ruegsegger 9].

The bone fraction, BV/TV, showed the strongest correlation despite the large overestimation. The overestimation is again a product of the partial volume effect associated with low resolution.

The MIL parameters show that the each parameter has a relatively strong correlation to the reference. However, the absolute values are not equally 
overestimated in the three directions. The lowest correlation between clinical CT and micro-CT is seen for MILz, probably due to the large slice thickness. This precludes meaningful comparisons between the different directions.

In conclusion, the high correlation for the parameters describing trabecular separation, trabecular number and bone volume fraction indicate that it might be possible to monitor changes in the microarchitecture of trabecular bone with clinical CT, as a complement to measurements of bone mineral density. The results would probably improve if a smaller slice thickness and a lower noise level could be produced.

\section{References}

1. Cullum, I.D., Ell, P.J., Ryder, J.P.: X-ray dual-photon absorptiometry: a new method for the measurement of bone density. Br J Radiol 62(739) (1989) 58792 Journal Article.

2. Parfitt, A.M., Drezner, M.K., Glorieux, F.H., Kanis, J.A., Malluche, H., Meunier, P.J., Ott, S.M., Recker, R.R.: Bone histomorphometry: standardization of nomenclature, symbols, and units. Report of the ASBMR Histomorphometry Nomenclature Committee. J Bone Miner Res 2(6) (1987) 595-610

3. Revol-Muller, C., Peyrin, F., Carrillon, Y., Odet, C.: Automated 3D region growing algorithm based on an assessment function. Pattern Recognition Letters 23(1-3) (2002) 137-150

4. Revol-Muller, C., Jourlin, M.: A new minimum variance region growing algorithm for image segmentation. Pattern Recognition Letters 18(3) (1997) 249-258

5. Xie, W., Thompson, R.P., Perucchio, R.: A topology-preserving parallel 3D thinning algorithm for extracting the curve skeleton. Pattern Recognition 36(7) (2003) 1529 1544

6. Borgefors, G.: On digital distance transforms in three dimensions. Computer Vision and Image Understanding 64(3) (1996) 368-376

7. Svensson, S., di Baja, G.S.: Using distance transforms to decompose 3D discrete objects. Image Vision Comput 20(8) (2002) 529-540

8. Hildebrand, T., Ruegsegger, P.: A new method for the model-independent assessment of thicness in three-dimensional images. Journal of Microscopy 185(1) (1997) $67-75$

9. Laib, A., Ruegsegger, P.: Comparison of structure extraction methods for in vivo trabecular bone measurements. Comput Med Imaging Graph 23(2) (1999) 69-74 